EXTENDED REPORT

\title{
Gout epidemiology: results from the UK General Practice Research Database, 1990-1999
}

\author{
T R Mikuls, J T Farrar, W B Bilker, S Fernandes, H R Schumacher Jr, K G Saag
}

Ann Rheum Dis 2005;64:267-272. doi: 10.1136/ard.2004.024091

See end of article for authors' affiliations

.....................

Correspondence to: Dr K G Saag, Department of Medicine, Division of Clinical Immunology and Rheumatology, 510 20th

Street South, FOT 8th

Floor, Birmingham, AL 35294, USA;

ksaag@uab.edu

Accepted 16 July 2004

\begin{abstract}
Objective: To examine the epidemiology of gout and gout treatment in the United Kingdom using a large national practice based population.

Methods: Data from the UK General Practice Research Database from 1990 to 1999 were examined. Physician diagnoses and drug codes were used, and trends in gout incidence and treatment examined. Additionally, disease prevalence for the year 1999 was assessed. To examine the association of gout with comorbid disease, the prevalence of select health conditions and drug use was compared with the corresponding prevalences seen in osteoarthritis, adjusting for both age and sex.

Results: From 1 January 1990 to 31 December 1999 overall gout incidence remained relatively stable, ranging from a low of 11.9 cases (95\% confidence interval (CI) 11.5 to 12.3 ) in 1991 to a high of 18.0 cases (95\% Cl 17.6 to 18.4) per 10000 patient-years in 1994 . Gout prevalence in 1999 was $1.4 \%$ with rates approaching $7 \%$ in men over the age of 65 . Drugs used for the treatment of gout remained constant in prevalent cases with the exception of a significant decline in non-steroidal anti-inflammatory drug use over the 10 year follow up. Compared with patients with osteoarthritis, patients with gout were significantly more likely to have cardiovascular disease, hypertension, diabetes, and chronic renal failure, and were more likely to have used diuretics or ciclosporin, or both.

Conclusion: Although gout is common in the UK, particularly among older men, the incidence of the disease seems to have remained stable during the 1990s.
\end{abstract}

$\mathrm{R}$ ecent reports suggest that the worldwide incidence and prevalence of gout are rising. Separate groups from both the USA $^{1}$ and New Zealand ${ }^{2}$ have observed an approximately twofold increase in gout incidence over the past 30 years. Harris and colleagues observed a threefold increase in gout prevalence in the UK over just two decades of follow up. ${ }^{34}$ Given the frequent association of gout with comorbid diseases, including hypertension, diabetes, and cardiovascular disease, ${ }^{5}$ a rising frequency of gout could have substantial public health implications.

To assess changing trends in gout incidence and the use of gout related treatments, we examined the epidemiology of gout and gout treatment in the General Practice Research Database (GPRD) of the UK (formerly the Value Added Medical Products Database). We expected that there would be an increase in overall gout incidence and an increase in the use of disease-specific drugs among patients with gout from 1990 to 1999. To examine the association of gout with comorbid disease, we also compared the prevalence of selected health conditions found in patients with gout with the corresponding prevalences seen in osteoarthritis, a chronic form of arthritis that like gout is more common among the elderly.

\section{METHODS}

\section{General Practice Research Database (GPRD)}

For the current study we examined data from the UK GPRD. The GPRD is a large population based database that currently collects data from participating general practices, which together in 1999 provided primary health care for over 1.8 million people in the UK ( $\sim 3 \%$ of the population). The GPRD falls under the jurisdiction of the UK Department of Health and is administered by the Office of National Statistics. ${ }^{6}$ The GPRD is among the most widely used data sources for pharmacoepidemiological research. ${ }^{7}$
The GPRD contains computerised health data that is directly entered by participating general practitioners after a mandatory trial period of data entry to ensure the accuracy and completeness of the data. ${ }^{7}$ Previous validation studies of the GPRD have shown that the recording of pertinent clinical data is highly accurate and nearly complete. ${ }^{89}$ The geographical distribution of GPRD patients is similar to that of the general UK population and recent comparisons of age and sex distribution have shown that the GPRD population is similar to those recorded in the UK National Population Census. ${ }^{7}$ The GPRD has been used to study a variety of clinical outcomes, including acute liver injury, gastrointestinal bleeding and peptic ulcerations, myocardial infarction, venous thromboembolism, neoplasia, seizure disorders, blood dyscrasias, and inflammatory bowel disease. ${ }^{70}$ Moreover, numerous studies have validated the accuracy of coded diagnoses recorded in the GPRD. ${ }^{7}$ Approval for this research was provided by the Office of National Statistics Scientific and Ethical Advisory Group and the Institutional Review Boards at the University of Alabama at Birmingham, the University of Pennsylvania, and the University of Nebraska.

\section{Case definition and data abstraction}

Clinical diagnoses were recorded using the Oxmis (Oxford Medical Information Systems) coding system. ${ }^{11}$ We searched the GPRD for all patients with a coded diagnosis of gout from 1 January 1990 to 31 December 1999. The following patient characteristics were abstracted for each prevalent case: age, sex, the presence or absence of select comorbid conditions (coronary artery disease, hypertension, diabetes mellitus, nephrolithiasis, and chronic renal failure), and concomitant drug use within the same calendar year (diuretics,

Abbreviations: $\mathrm{Cl}$, confidence interval; GPRD, General Practice Research Database; NSAIDs, non-steroidal anti-inflammatory drugs; $\mathrm{OR}$, odds ratio 

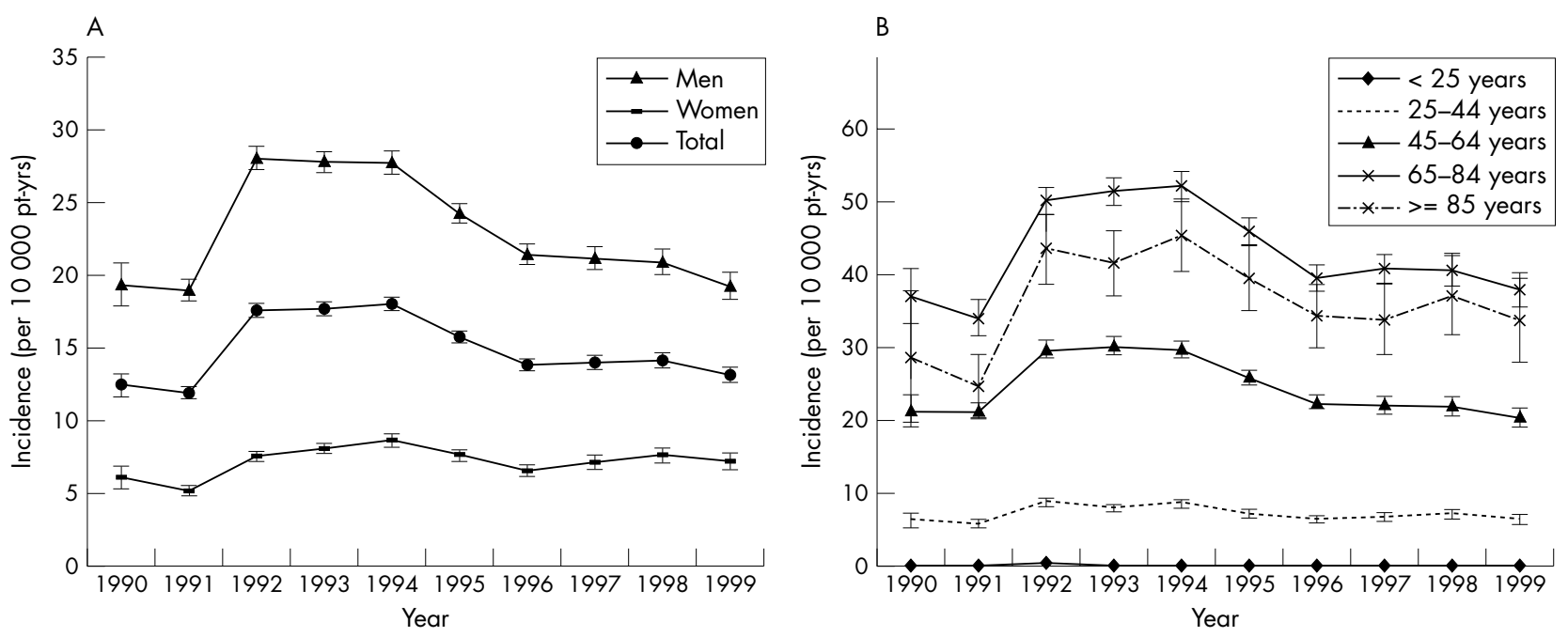

Figure 1 (A) Overall and sex-specific annual gout incidence (1990-99) among enrolees in the UK GPRD. (B) Annual UK gout incidence (1990-99) by age distribution. $95 \% \mathrm{Cls}$ (shown with bars) calculated using normal approximation.

ciclosporin, urate lowering drugs, colchicine, non-steroidal anti-inflammatory drugs (NSAIDs), and glucocorticoids).

Incident cases included those with a minimum of 12 months of research standard data available in the database before an initial gout diagnosis. We also examined the frequency with which gout related medicines (that is, NSAIDs) were prescribed among incident cases (before and up to 30 days after the incident date). Similar data, with the exception of gout-specific medicine use (that is, allopurinol, colchicine), were abstracted from the records of the remaining GPRD patients without gout who had a diagnosis of osteoarthritis.

\section{Analyses}

Gout incidence rates (per 10000 patient-years) were calculated for each calendar year from 1 January 1990 to 31 December 1999. We limited the calculations of annual incidence to the 10 year time period after 1 January 1990 because before this date, the GPRD did not contain the complete medical records necessary for the calculation of incidence estimates. ${ }^{7}$ To facilitate comparisons with prior national studies from the UK that have reported a 1 year period prevalence, ${ }^{3}{ }^{4}$ we also calculated a period prevalence for the 1999 calendar year. Prevalence estimates were limited to 1999 because data on prevalent conditions (those present at time of enrolment into the GPRD) were not complete in the early 1990s. ${ }^{12}$ Confidence intervals were determined for all incidence and prevalence estimates using normal approximation. ${ }^{13}$ All analyses were conducted using SAS (SAS Institute, Cary, NC) and Stata (Stata Corporation, College Station, TX).

Logistic regression with patients with osteoarthritis as the referent group was used, and age and sex adjusted odds ratios (ORs) and 95\% confidence intervals (CIs) were calculated to describe the association of gout with select patient sociodemographic and health characteristics. Group comparisons of the total number of concomitant drugs used were completed using Poisson regression. Patients with an overlapping diagnosis of both gout and osteoarthritis were removed from the analyses. Trends in the annual prevalence of gout drug use from 1990 to 1999 were examined using the Cochran-Armitage trend test.

Table 1 Sex- and age-specific gout incidence in the UK GPRD, 1999

\begin{tabular}{|c|c|c|c|c|}
\hline Age (years) & $\begin{array}{l}\text { Cases } \\
\text { (n) }\end{array}$ & $\begin{array}{l}\text { At risk } \\
\text { population } \\
\text { (n) }\end{array}$ & $\begin{array}{l}\text { Incidence } \\
\text { (per } 10000 \\
\text { patient-years) }\end{array}$ & $95 \% \mathrm{Cl}$ \\
\hline \multicolumn{5}{|l|}{ Men } \\
\hline$<25$ & 12 & 255950 & 0.5 & 0.2 to 0.7 \\
\hline $25-44$ & 293 & 261406 & 11.2 & 9.9 to 12.5 \\
\hline $45-64$ & 694 & 209642 & 33.1 & 30.7 to 35.5 \\
\hline $65-84$ & 576 & 107941 & 53.4 & 49.0 to 57.8 \\
\hline$>85$ & 56 & 11246 & 49.8 & 36.8 to 62.8 \\
\hline \multicolumn{5}{|l|}{ Women } \\
\hline$<25$ & 1 & 246346 & 0.04 & 0.0 to 0.1 \\
\hline $25-44$ & 41 & 254958 & 1.6 & 1.1 to 2.1 \\
\hline $45-64$ & 157 & 205382 & 7.6 & 6.4 to 8.8 \\
\hline $65-84$ & 348 & 135305 & 25.7 & 23.0 to 28.4 \\
\hline$>85$ & 77 & 28100 & 27.4 & 21.3 to 33.5 \\
\hline \multicolumn{5}{|l|}{ Total } \\
\hline$<25$ & 13 & 502296 & 0.3 & 0.1 to 0.4 \\
\hline $25-44$ & 334 & 516364 & 6.5 & 5.8 to 7.2 \\
\hline $45-64$ & 851 & 415024 & 20.5 & 19.1 to 21.9 \\
\hline $65-84$ & 924 & 243246 & 38.0 & 35.5 to 40.3 \\
\hline$>85$ & 133 & 39346 & 33.8 & 28.1 to 39.5 \\
\hline
\end{tabular}




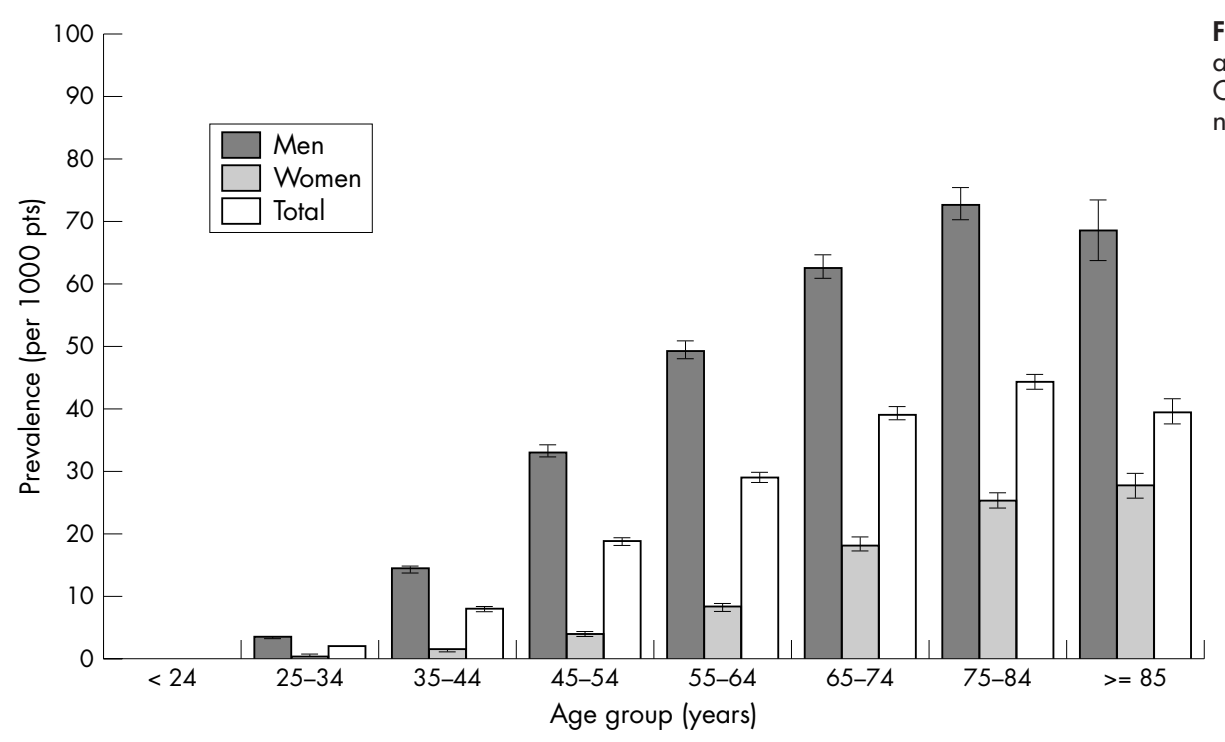

Figure 2 Gout prevalence (1999) among enrolees in the UK GPRD. 95\% $\mathrm{Cls}$ (shown with bars) calculated using normal approximation.

\section{RESULTS}

\section{Incidence}

From 1 January 1990 to 31 December 1999 overall gout incidence remained relatively stable with the exception that rates were slightly higher from 1992 to 1994 (fig 1). Overall gout incidence ranged from a low in 1991 of 11.9 cases $(95 \%$ CI 11.5 to 12.3 ) to a high of 18.0 cases ( $95 \%$ CI 17.6 to 18.4 ) in 1994, before declining to 13.1 cases (95\% CI 12.6 to 13.7 ) per 10000 patient-years in 1999. Overall, the highest gout incidence was consistently observed among older men while the lowest rates were seen in younger women. In 1999 the highest disease incidence was found in men aged 65-84 years (53.4 cases per 10000 patient-years; $95 \%$ CI 49.0 to 57.8; table 1). In contrast, gout was very rare in women below the age of 45 (0.8 cases per 10000 patient-years; 95\% CI 0.5 to 1.1). Figure 1 summarises the annual incidence by sex and age distribution.

\section{Prevalence}

During the 1999 calendar year, there were approximately 1.7 million patients $(49 \%$ male) with up to standard data available in the GPRD. During this year, 23918 patients had a diagnosis of gout, resulting in an overall prevalence of
$1.39 \%$ (95\% CI 1.37 to 1.41 ). The overall male to female ratio for prevalent cases was 3.6:1. Figure 2 summarises gout prevalence for 1999, stratified by age and sex. Prevalence rates peaked in men between the ages of 75 and 84 years $(7.3 \%)$ while in women disease prevalence continued to rise beyond 85 years of age $(2.8 \%)$.

\section{Sociodemographic and disease factors in gout and osteoarthritis}

From 1 January 1990 to 31 December 1999 there were a total of 63105 GPRD enrolees with a diagnosis of gout compared with 157489 enrolees with osteoarthritis. This included 6622 patients with overlapping diagnoses of both gout and osteoarthritis, who were excluded from the analyses. Table 2 summarises the demographic and health characteristics of the two patient groups. Patients with gout were younger than patients with osteoarthritis $(\mathrm{p}<0.001)$ and were more commonly male $(\mathrm{p}<0.001)$. The patient groups had an identical duration of follow up in the database (mean (SD) 3.7 (2.8) years).

After adjusting for both age and sex, patients with gout were significantly more likely than patients with osteoarthritis to have coronary artery disease, hypertension,

Table 2 Sociodemographic and health related characteristics of patients with gout and osteoarthritis: the UK General Practice Research Database (GPRD), 1990-99

\begin{tabular}{|c|c|c|c|c|c|c|}
\hline $\begin{array}{l}\text { Patients' characteristic/disease } \\
\text { related factor }\end{array}$ & $\begin{array}{l}\text { Patients with gout } \\
(\mathrm{n}=56483 \text { ) }\end{array}$ & $\begin{array}{l}\text { Patients with OA } \\
(\mathrm{n}=150867)\end{array}$ & $\begin{array}{l}\text { Crude OR } \\
\text { (gout } v \text { OA) }\end{array}$ & $95 \% \mathrm{Cl}$ & $\begin{array}{l}\text { Adjusted } \dagger \text { OR } \\
\text { (gout } v \text { OA) }\end{array}$ & $95 \% \mathrm{Cl}$ \\
\hline \multicolumn{7}{|l|}{ Sociodemographic } \\
\hline Age (years), mean $(S D)^{*}$ & $60.5(15.4)$ & $66.8(13.4)$ & - & - & & \\
\hline Sex (male) & $44959(79.6)$ & $51019(33.8)$ & 7.64 & 7.46 to 7.81 & - & - \\
\hline \multicolumn{7}{|l|}{ Comorbidity } \\
\hline Coronary artery disease & 14039 (24.9) & 29855 (19.8) & 1.34 & 1.31 to 1.37 & 1.75 & 1.70 to 1.79 \\
\hline Hypertension & $13483(23.9)$ & $30714(20.4)$ & 1.23 & 1.20 to 1.26 & 1.52 & 1.48 to 1.56 \\
\hline Diabetes mellitus & $3632(6.4)$ & $8995(6.0)$ & 1.08 & 1.04 to 1.13 & 1.11 & 1.06 to 1.16 \\
\hline Nephrolithiasis & $506(0.9)$ & $996(0.7)$ & 1.36 & 1.22 to 1.52 & 0.87 & 0.77 to 0.97 \\
\hline Chronic renal failure & $565(1.0)$ & $365(0.2)$ & 4.17 & 3.65 to 4.75 & 4.95 & 4.28 to 5.72 \\
\hline Renal transplant & $103(0.2)$ & $11(0.0)$ & 25.05 & 13.45 to 46.66 & 25.13 & 12.97 to 48.68 \\
\hline \multicolumn{7}{|l|}{ Concomitant drug use } \\
\hline Diuretics & $18392(32.6)$ & $51366(34.0)$ & 0.94 & 0.92 to 0.96 & 1.72 & 1.67 to 1.76 \\
\hline Ciclosporin or FK-506 & $258(0.5)$ & $80(0.1)$ & 8.65 & 6.73 to 11.12 & 7.93 & 5.97 to 10.54 \\
\hline
\end{tabular}

${ }^{*} p<0.001$ for difference; tadjusted for age and sex.

Results are shown as No (\%) unless otherwise indicated. 


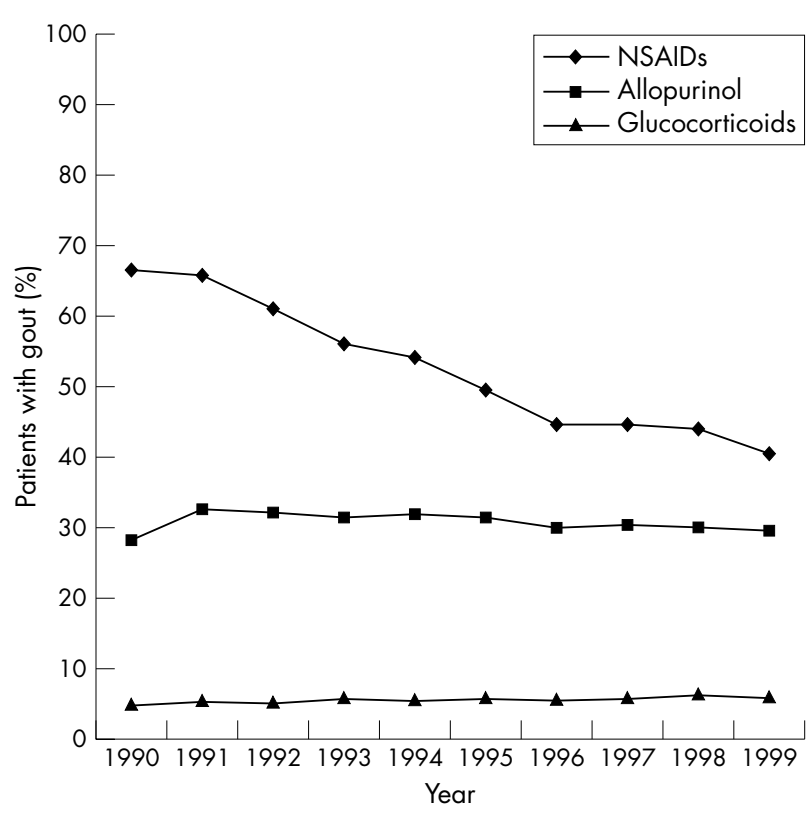

Figure 3 Annual frequency of NSAID, allopurinol, and glucocorticoid use among prevalent patients with gout $(n=63105)$ enrolled in the UK GPRD, 1990-1999; frequency of use includes any prescription received within the same calendar year. Colchicine (range 0.9-3.4\%) and uricosuric (range $0.8-1.0 \%$ ) use are not shown. $p$ Value $<0.001$ for trend for NSAID use among prevalent patients with gout. NSAID use among prevalent patients with osteoarthritis also declined during the period of observation (data not shown) from a high of $63.7 \%$ in 1990 to a low of $39.0 \%$ in 1999 ( $p<0.001$ for trend).

diabetes, chronic renal failure, and were prescribed a greater number of total drugs (mean (SD) 1.9 (2.3) v 0.7 (0.5) drugs; $\mathrm{p}<0.001)$. Compared with patients with osteoarthritis, patients with gout were also substantially more likely to have a history of renal transplantation, in addition to the use of diuretics or ciclosporin. Although patients with gout were more likely ( $\mathrm{OR}=1.36$; $95 \%$ CI 1.22 to 1.52$)$ than patients with osteoarthritis to have a history of nephrolithiasis in our unadjusted analysis, this effect was reversed after controlling for age and sex. Compared with a diagnosis of osteoarthritis, gout was actually associated with an approximately $10 \%$ lower odds of having nephrolithiasis ( OR $=0.87$; 95\% CI 0.77 to 0.97 ) after accounting for the effects of both age and sex. Results of our age and sex adjusted analyses were not changed after controlling for multiple comorbidities, drug use, and duration of follow up (data not shown).

\section{Trends in drug use}

From 1990 to 1999, the annual frequency of allopurinol (range 25.3-29.5\%) and glucocorticoid (6.4-9.6\%) use among all patients with gout remained stable (fig 3). Although less often prescribed, the use of uricosuric drugs $(0.8-1.0 \%)$ and colchicine (0.9-3.4\%) also remained constant over time (data not shown). In contrast, there was a significant decrease in annual NSAID use among prevalent GPRD patients with gout, declining steadily from a rate of $66.5 \%$ in 1990 to a low of $40.6 \%$ in $1999(\mathrm{p}<0.001$ for trend $)$. During the same interval (1990-1999), there was a similar decline in NSAID use among prevalent osteoarthritis cases, falling from a high of $63.7 \%$ in 1990 to just $39.0 \%$ in 1999 ( $p<0.001$ for trend). When we restricted our analysis to incident cases, we found that NSAID use (before and up to 30 days after the incident date) remained stable over the same period of follow up (range 86.7-89.9\%) (data not shown).

\section{DISCUSSION}

We did not observe a consistent increase in disease incidence over the 10 year time interval beginning in 1990 nor did we observe increases in the frequency of gout related drug use. On the contrary, we observed a significant decline in NSAID use among prevalent GPRD patients with gout between 1990 and 1999. In this practice based study, we observed an overall gout prevalence of $1.4 \%$ in 1999 . Corroborating previous epidemiological investigations, ${ }^{414}$ we found the highest prevalence estimates among elderly men, with rates approaching $7 \%$ in men over the age of 65 . Our comparison with patients with osteoarthritis showed that patients with gout were younger, more often male and more frequently had comorbidity and concomitant drug use.

Although the national incidence of gout has been investigated in both the UK ${ }^{15}$ and the USA, ${ }^{16-20}$ representative UK data on gout are relatively scarce, and no UK studies of gout have been conducted in the past decade. In a 1981 report, the annual gout incidence in the UK was 14 cases per $10000,{ }^{15}$ similar to the overall incidence found in our study (11.9-19.0 cases per 10000 patient-years). Estimates from the UK, including those from our study, are slightly higher than those seen in American studies. In a report from the Rochester Epidemiology Project investigators observed an overall age and sex adjusted incidence rate of 6.2 cases per $10000 .{ }^{1}$ Investigators from the Framingham Study found an overall gout incidence of 8.4 cases per $10000 .{ }^{18}$ Similarly, the 1999 male:female incidence ratio in our study is similar to that previously reported from the $\mathrm{UK}^{15}$ but lower than that seen in the USA. ${ }^{18}$

The perceived differences in gout incidence observed in studies from the UK and those done in the USA may relate to differences in the populations studied, or methods used in defining gout status. The sex ratio observed in our study, for instance, appears to be related to a relatively higher incidence of gout in older women. We found an incidence among older women that was approximately twice that seen in the early 1980s. ${ }^{15}$ Studies from the UK, including ours, have defined gout case status using physician diagnosis. In contrast, American studies have used biennial examinations, ${ }^{18}$ self report by physicians, ${ }^{20}$ or the 1977 American College of Rheumatology criteria ${ }^{1}$ for gout validation. ${ }^{21}$ These different methods for gout case ascertainment have various strengths and limitations. Although physical examinations of patients are optimal, this approach is limited by the periodic nature of gout, resulting in potentially inaccurate recall and added cost and inconvenience.

The 1999 gout prevalence observed in our study (1.4\%) is higher than that reported in the most recent national UK study. ${ }^{4}$ Based on our results, it can be estimated that there are currently over 700000 people with gout in the UK alone, one third of whom are receiving active treatment with urate lowering drugs. Harris's data from 40 volunteer practices in 1993, found gout prevalence to be 9.5 cases per 1000 patients, ${ }^{4}$ an estimate that is close to our finding and nearly three times higher than that seen in a similar national study conducted in the mid-1970s (2.6 cases per 1000 patients). ${ }^{3}$ Even if we were to restrict our case definition to include only patients with a gout diagnosis receiving allopurinol, the 1999 gout prevalence (4.1 cases per 1000 patients) would still be nearly twice that reported by Currie. If we assume that our estimates are accurate, these data suggest that effective preventive strategies are markedly underused in the treatment of gout.

There are potential explanations for the differences in gout prevalence in our study compared with those reported in earlier UK investigations. Although variations in methodology limit comparisons that can be made across studies, results of other investigations suggest that the apparent rise 
in gout prevalence is due predominantly to an increase in disease incidence. ${ }^{12}$ Although rates of obesity and diuretic use (known gout risk factors) have increased over time, our results do not support the contention that disease prevalence has increased simply as a function of incidence, at least during the 1990s. Because disease prevalence is a product of both incidence and disease duration, our observation of a relatively stable disease incidence over the past decade lends support to the notion that advancing longevity is a primary factor in increasing disease prevalence.

Our observation of decreased NSAID use among patients with gout from 1990 to 1999 was not expected. Given a similar temporal trend in NSAID use among patients with osteoarthritis, this decline does not appear to be unique to gout. It may be that with greater disease duration patients with both gout and osteoarthritis increasingly choose to treat themselves with over the counter NSAIDs, which became available in the UK in 1983. Our observation of stable NSAID prescribing over time among incident cases further suggests that the decline in NSAID use among prevalent gout cases does not reflect changes in physician prescribing patterns.

The association of gout with selected comorbidities, including cardiovascular disease, hypertension, and diabetes, is highly consistent with previous reports. Although it is recognised that comorbid conditions (for example, hypertension and renal insufficiency) contribute to gout incidence, it is becoming increasingly clear that gout and hyperuricaemia may have a causative role in comorbid disease. Hyperuricaemia has recently been linked to an increased risk for both ischaemic heart disease ${ }^{22}$ and cardiovascular mortality. ${ }^{23}$ Alternatively, hypertension has long been recognised as a risk factor for gout. ${ }^{54}$ In a recent study, the incidence of gout has been shown to be significantly increased among hypertensive patients even in the absence of diuretic treatment. ${ }^{24}$ Although the association between gout and diabetes is less clearly defined, obesity and insulin resistance may represent important links between these two conditions. Increased body weight is associated with higher serum urate levels and an increased risk of gout. ${ }^{17} 2025$ Obesity also leads to insulin resistance, which in turn results in an enhanced risk for the development of type II diabetes mellitus and cardiovascular morbidity. ${ }^{26}$

The lower odds of nephrolithiasis that we found associated with gout appear to be contrary to past studies that have shown an increased risk of kidney stones associated with hyperuricaemia ${ }^{27}$ and gout. ${ }^{28} 29$ The difference between our findings and those of previous investigations may relate to our choice of comparator group, which included elderly adults with substantial comorbid disease and concomitant drug use.

There are limitations to this study. As in other national investigations that have examined gout incidence and prevalence in the UK, ${ }^{3}{ }^{4}$ we relied on physician diagnoses to define case status, a definition that is subject to misclassification. The most specific epidemiological definition would require documentation of urate crystals in joint fluid to define gout incidence, but this would result in a definition that is very insensitive because the procedure is seldom used in primary care. ${ }^{1}$ Given the validated accuracy of the GPRD for several different incident health conditions, we do not believe that our incidence estimates are biased by a significant proportion of misdiagnoses. The GPRD, for instance, has been shown to be highly valid for the diagnosis of inflammatory bowel disease, ${ }^{10}$ a condition, like gout, that is marked by intermittent bouts of inflammation.

Disease prevalence estimates are less robust than disease incidence because prevalence estimates require conditions to be documented at the time of patient entry into the GPRD. Possibly, incomplete coding of pre-existing conditions might have led to prevalent gout cases being misclassified as incident cases. This may in part explain the increased disease incidence observed from 1992 to 1994, a period of substantial patient influx into the GPRD. To minimise the possible misclassification of prevalent gout cases, we required that incident cases have a minimum of 1 year of research standard data in the database before an initial diagnosis. Lengthening this time period up to 2 years did not substantially alter our results (data not shown).

Because the GPRD is practice based, it is possible that we missed patients with less severe gout who failed to present to their physician, suggesting that we may have underestimated the true population based incidence and prevalence of gout. Owing to limitations of the GPRD dataset, we were also unable to examine the associations of gout with other important variables, including socioeconomic status, education, race/ethnicity, body weight, or serum urate levels.

Current projections show that the proportion of people in the population aged 65 or older will grow more than threefold in the next 30 years alone. If it is assumed that its prevalence remains stable or increases in the elderly, gout will become an increasingly recognised source of morbidity. These projections, coupled with its known associations with concomitant polypharmacy and frequently inappropriate drug use, ${ }^{30}$ highlight the fact that gout and its comorbidities are increasingly important public health concerns.

\section{Authors' affiliations}

T R Mikuls, Department of Medicine, University of Nebraska Medical Center, and Omaha VA Medical Center, Omaha, NE, USA

J T Farrar, W B Bilker, S Fernandes, Center for Clinical Epidemiology and Biostatistics and Center for Education and Research in Therapeutics (CERT), University of Pennsylvania, USA

H R SchumacherJr, Philadelphia VA Medical Center, Philadelphia, PA, USA

K G Saag, Department of Medicine and Center for Education and Research in Therapeutics (CERT) in Musculoskeletal Disorders, University of Alabama at Birmingham, Birmingham, AL, USA

\section{REFERENCES}

1 Aromdee E, Michet C, Crowson C, O'Fallon M, Gabriel S. Epidemiology of gout: is the incidence rising? J Rheumatol 2002;29:2403-6.

2 Klemp P, Stansfield S, Castle B, Robertson M. Gout is on the increase in New Zealand. Ann Rheum Dis 1997;56:22-6.

3 Currie W. Prevalence and incidence of the diagnosis of gout in Great Britain. Ann Rheum Dis 1979;38:101-6.

4 Harris C, Lloyd C, Lewis J. The prevalence and prophylaxis of gout in England. $J$ Clin Epidemiol 1995;48:1153-8.

5 Hochberg M. Gout. In: Silman A, Hochberg M, eds. Epidemiology of the rheumatic diseases. New York: Oxford University Press, 2001:230-46.

6 Hollowell J. The General Practice Research Database: quality of morbidity data. Population Trends 1997;87:36-40.

7 Garcia Rodriguez L, Perez Gutthann S. Use of the UK General Practice Research Database for pharmacoepidemiology. Br J Clin Pharmacol 1998;45:419-25.

8 Jick H, Jick S, Derby L. Validation of information recorded on general practitioner based computerised data resource in the United Kingdom. BMJ 1991;302:766-8.

9 Jick H, Terris B, Derby L, Jick S. Further validation of information recorded on a general practitioner based computerised data resource in the United Kingdom. Pharmacoepidemiol Drug Safety 1992;1:347-9.

10 Lewis J, Brensinger C, Bilker W, Strom B. Validity and completeness of the General Practice Research Database for studies of inflammatory bowel disease. Pharmacoepidemiol Drug Safe 2002;11:211-18.

11 Perry J. Oxmis problem code for primary medical care. Oxford: Oxmis Publications, 1971.

12 Farrar J, Mikuls T, Saag K, Bilker W, Fernandes S, Strom B. Issues in the determination of disease prevalence from medical record databases: cumulative incidence in chronic diseases such as gout. 19th International Conference on Pharmacoepidemiology. Philadelphia, 2003.

13 Ahlbom A. Biostatistics for epidemiologists. Boca Raton: Lewis Publishers, 1993.

14 Benson V, Marano M. Current estimates from the National Health Interview Survey, 1992. Vital Health Stat 1992;189:1-269.

15 Stewart O, Silman A. Review of UK data on the rheumatic diseases-4 Gout. Br J Rheumatol 1990;29:485-8.

16 Hall A, Barry P, Dawber T, McNamara P. Epidemiology of gout and hyperuricemia: a long-term population study. Am J Med 1967;42:27-37. 
17 Campion E, Glynn R, DeLabry L. Asymptomatic hyperuricemia. Risks and consequences in the Normative Aging Study. Am J Med 1987:82:421-6

18 Abbott R, Brand F, Kannel W, Castelli W. Gout and coronary heart disease: the Framingham Study. J Clin Epidemiol 1988;41:237-242.

19 Hochberg M, Thomas J, Thomas D, Mead L, Levine D, Klag M. Racial differences in the incidence of gout: the role of hypertension. Arthritis Rheum 1995:38:628-32.

20 Roubenoff R, Klag M, Mead L, Liang K, Seidler A, Hochberg M. Incidence and risk factors for gout in white men. JAMA 1991;266:3004-7.

21 Wallace S, Robinson H, Masi A, Decker J, McCarty D, Yu T-F. Preliminary criteria for the classification of the acute arthritis of primary gout. Arthritis Rheum 1977;20:895-900.

22 Freedman D, Williamson D, Gunter E, Byers T. Relation of serum uric acid to mortality and ischemic heart disease. The NHANES I Epidemiologic Follow-up Study. Am J Epidemiol 1995;141:637-44.

23 Fang J, Alderman M. Serum uric acid and cardiovascular mortality the NHANES I epidemiologic follow-up study, 1971-1992. National Health and Nutrition Examination Survey. JAMA 2000;283:2404-10.
24 Grodzicki T, Palmer A, Bulpitt C. Incidence of diabetes and gout in hypertensive patients during 8 years of follow-up. The General Practice Hypertension Study Group. J Hum Hypertens 1997; 11:583-5.

25 Lin K, Lin H, Chou P. The interaction between uric acid level and other risk factors on the development of gout among asymptomatic hyperuricemic men in a prospective study. J Rheumatol 2000;27:1501-5.

26 Haffner $\mathbf{S}$. Pre-diabetes, insulin resistance, inflammation and CVD risk. Diabetes Res Clin Pract 2003;61(suppl):S9-18.

27 Halabe A, Sperling O. Uric acid nephrolithiasis. Miner Electrolyte Metab 1994;20:424-31.

28 Kramer H, Choi H, Atkinson K, Stampfer M, Curhan G. The association between gout and nephrolithiasis in men: the Health Professionals' Follow-up Study. Kidney Int 2003;64:1022-6.

29 Kramer H, Curhan G. The association between gout and nephrolithiasis: the National Health and Nutrition Examination Survey III, 1988-1994. Am J Kidney Dis 2002;40:37-42.

30 Chin M, Wang L, Jin L, Mulliken R, Walter J, Hayley DC, et al. Appropriateness of medication selection for older persons in an urban academic emergency department. Acad Emerg Med 1999;6:1232-42. 